\title{
Identifying Differences in Elastographic Properties of Calf Muscles and Tendons Across Subsets of Tennis Players
}

\author{
C. D. Sacks', R. A. Gallo², L. Kong ${ }^{3}$, D. H. Cortes $^{4}$ \\ ${ }^{1}$ College of Medicine, The Pennsylvania State University, Hershey, Pennsylvania, U.S.A. \\ 2 Department of Orthopedics and Rehabilitation, College of Medicine, The Pennsylvania State University, \\ Hershey, Pennsylvania, U.S.A. \\ ${ }^{3}$ Division of Biostatistics and Bioinformatics, Department of Public Health Sciences, College of Medicine, The \\ Pennsylvania State University, Hershey, Pennsylvania, U.S.A. \\ ${ }^{4}$ Department of Mechanical and Nuclear Engineering, The Pennsylvania State University, University Park, \\ Pennsylvania, U.S.A.
}

\section{CORRESPONDING AUTHOR: \\ Robert A. Gallo \\ Department of Orthopedics \\ and Rehabilitation \\ College of Medicine \\ The Pennsylvania State University \\ 30 Hope Drive \\ Hershey PA 17033, U.S.A. \\ E-mail: rgallo@pennstatehealth.psu.edu}

DOI:

10.32098/mltj.02.2021.12

LEVEL OF EVIDENCE: 5

\section{SUMMARY}

Objective. Calf strain occurs frequently in tennis players and has been termed "tennis leg". To date, there is a lack of information showing how injury history, age, gender, or level of play, may predispose tennis players to injury $(1,2)$. The purpose of this study was to investigate the elastographic properties of the gastrocnemius-soleus complex in a group of club-level tennis players. We hypothesized that elastographic properties of the gastrocnemius-complex are affected by age and sport specificity (tennis only $v s$ multi-sport).

Methods. Participants were recruited through contact personnel of a local tennis club. At the club's annual summer tournament, each participant voluntarily consented and completed a survey to obtain information about age, level of play, length of play, participation in other sports, relevant injuries, and relevant surgeries. Participants underwent ultrasonography that included visualization of the medial gastrocnemius musculotendinous junction, soleus muscle, and Achilles tendons, bilaterally. Shear wave speed (SWS) was gathered from ultrasonography for all tissues to provide a comparative standard unit of measurement. The data was analyzed with a mixed effects model. Results. 20 participants with mean age 33.5 years old (range, 14-61) were evaluated. There was a statistically significant trend in medial gastrocnemius SWS depending on age, participation in sports other than tennis, and two different metrics for skill level (highest level of competitive play and USTA NTRP rating). There was a statistically significant trend between Achilles tendon SWS and highest level of competitive play. Results showed no significant trends for any of the tissues and serving hand, whether the ultrasound was conducted before or after a match, or for any of the four metrics for tennis experience (times playing tennis per week, overall tennis experience, years playing $>3$ times per week, and whether a player had $>10$ or $<10$ years of experience). No statistically significant trends were observed for the soleus muscle when compared to any of the demographics.

Conclusions. Age, exclusively playing tennis as opposed to other sports, and two metrics of skill level (highest level of competitive play and USTA NTRP rating) significantly affected gastrocnemius SWS. Highest level of competitive play was the only metric found to affect Achilles tendon SWS. Soleus SWS exhibited no significant changes with any of the variables, despite following similar trends with gastrocnemius SWS.

\section{KEY WORDS}

Achilles tendon; calf strain; Gastrocnemius-Soleus Complex; tennis leg; tennis. 


\section{INTRODUCTION}

Tennis elbow, or lateral epicondylitis, is a well-known activity-related injury, but less discussed is tennis leg, or calfstrain. Like lateral epicondylitis, calf strain commonly occurs in tennis players, but also occurs in people who do not play tennis $(1,3)$. It was first described by Powell in 1883 as a tissue rupture at the distal and medial musculotendinous junction where the AT meets the gastrocnemius muscle (4). "Tennis leg" has been commonly described among tennis players and is most often seen in people between ages of 30-45. Risk factors that predispose this population to calf strain are unknown. Clinical presentation commonly involves a middle-aged patient who has acute activity-related pain in the medial portion of their calf, accompanied by a "popping" sound when stretching their gastrocnemius muscle through dorsiflexion of their foot (5). There are various stages of injury severity, ranging from minor structural injury to complete tear of the muscle from the tendon (6). Treatment is largely conservative, consisting of "RICE," or rest, ice, compression, and elevation, to promote tissue healing (5).

Ultrasound is an effective method of quantifying the elastographic properties of various tissue types and has previously been used in muscle and tendon studies of athletes such as volleyball players and distance runners, where cross-sectional area, elongation, and force were obtained and analyzed ( 7 , 8). In this study, ultrasound has been used to identify elastographic differences of the gastrocnemius-soleus complex across tennis players according to age, gender, level of play, and length of play. While it is observed that the injury occurs more often in middle-aged tennis players than younger tennis players or their non-tennis-playing counterparts, little is known about specific risk factors related to calf strain.

The objective of this study is to evaluate the elastographic properties of the Achilles tendon in club-level tennis players. We hypothesized that elastographic properties of the gastrocnemius-complex are affected by age and sport specificity (tennis only vs multi-sport).

\section{METHODS}

This study was ethically conducted according to the international standards described in (9). It was performed as a pilot study with a level of evidence of V. It was conducted at an academic medical center and approved by the Institutional Review Board according to Protocol for Human Subject Research (9). Once approval was obtained, permission from a local tennis club was granted to recruit participants and collect ultrasound data during the first two consecutive days during their 2019 Summer Tennis Tournament.

If they were willing to participate, the tennis players were first screened according to the inclusion and exclusion crite- ria. Inclusion criteria were competitive male and female tennis players with minimum age of 14 and under the age of 65 . Exclusion criteria were previous injury or surgery to the lower extremity. Once consent was obtained, each participant completed a survey pertaining to participant age, gender, level of play, length of play, participation in other sports, relevant injuries, and relevant surgeries. While in an unloaded plantarflexion position, participants underwent ultrasonography of their medial muscle-tendon junction of the Achilles tendon and gastrocnemius, bilaterally, either before or after partaking in their tennis match. This set-up is shown in figure 1. Excellent interobserver correlation, at several locations of the Achilles tendon, including the muscle-tendon junction, has been reported when the lower leg is in the unloaded plantar flexion position (10).

A Verasonic ultrasound system (Verasonic Inc., Redmond, WA, USA) with a L7-4 transducer (center frequency $=5.2080$ $\mathrm{MHz}$, and beam width $=4-7 \mathrm{MHz}$ ) was used. A customized supersonic shear wave elastography (SWE) method was used to measure shear modulus within lower leg muscles (11). The technique was validated using calibrated homogeneous elasticity phantom having various shear modulus values (Model 040GSE, CIRS, Norfolk, Virginia, USA) as well as other musculoskeletal tissues with the intraday and day-to-day reliability of 0.72 (95\% Confidence Interval (CI) $=0.59-0.83)$ and $(0.95$ with $95 \% \mathrm{CI}=0.88-0.98)$, respectively (12). The ROI size of $7.39 \mathrm{~mm} \times 7.39 \mathrm{~mm}$ was selected for the measurement of lower leg shear modulus in the gastrocnemius and soleus muscles, while the ROI height for the Achilles tendon measurements was adjusted to include just the tendon $($ ROI size $=$ Tendon thickness $\times 7.39 \mathrm{~mm})(13)$. After data collection, ultrasound data was analyzed to identify the elastographic properties of the Achilles tendon-gastrocnemius junction. Shear wave speed (SWS) was the primary measurement used to compare tissue properties between all three tissues. SWS was used to analyze muscle in addition to tendon to keep analysis consistent, instead of comparing tendon SWS to muscle shear modulus.

The analyzed data was subject to statistical analysis using a mixed effects model to identify any significant differences in Achilles tendon-gastrocnemius junction properties based on age, gender, level of play, and length of play. The effect of age on SWS was also analyzed by dividing the participants into two groups: young (under 35 years old) or older ( 35 years old or older). All tests were two-sided, with p-values $<$ 0.05 being considered statistically significant.

\section{RESULTS}

Twenty participants with a mean age 33.5 years (range, 14-61 years old) participated in this study. Table I depicts 

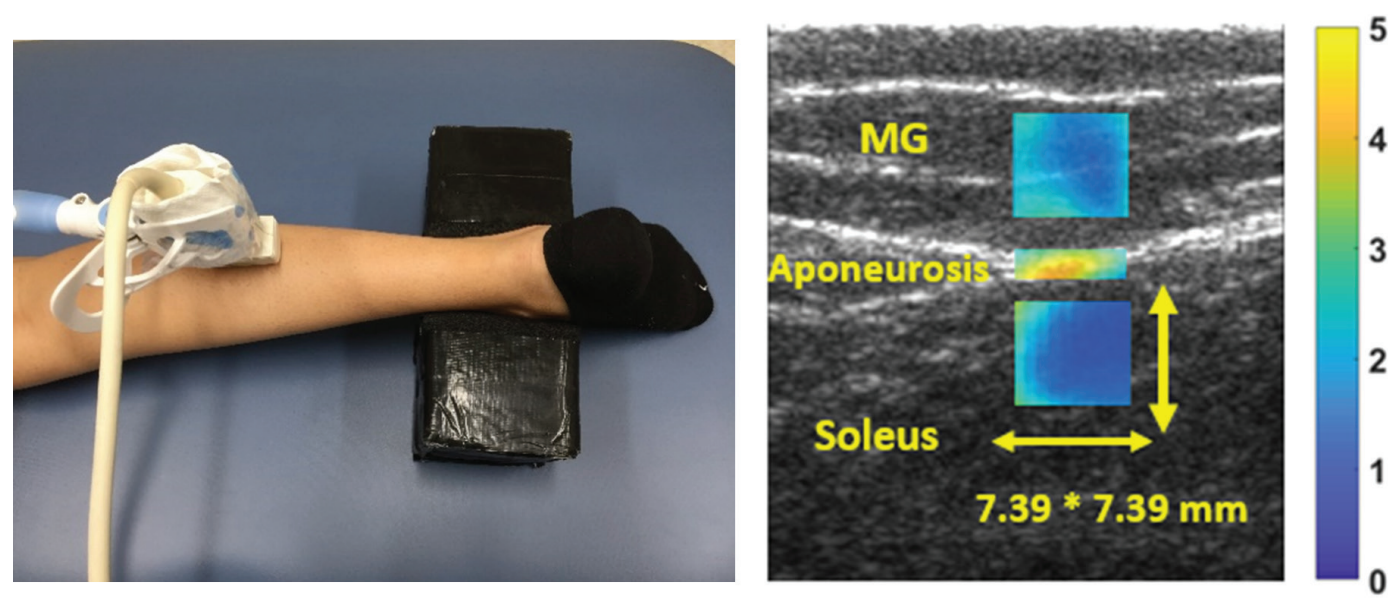

$5 \mathrm{~m} / \mathrm{s}$

4

3

2

1

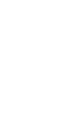

Figure 1. The physical setup of data collection and the image that the ultrasound probe generated. As shown on the left, the ultrasound probe was placed on the posterior lower extremity at the musculotendinous junction comprised by the Achilles tendon, medial gastrocnemius (MG), and soleus. These three structures are shown in the ultrasound image on the right. Shear wave speed (SWS) is depicted by the color gradient, ranging from 0-5 meters per second $(\mathrm{m} / \mathrm{s})$. A scale in millimeters (mm) is given for size reference.

SWS values obtained for all participants and illustrates each participant's tennis profile, including duration of experience, highest level of play, other sports played, and whether ultrasound scan occurred before or after a match. Table II organizes this data and states the highest, lowest, and average values for tissue SWS. Table III shows a summary of the significant and non-significant associations between the variables tested against gastrocnemius, soleus, and Achilles tendon SWS.

\section{Age}

There was a significant negative correlation between age and gastrocnemius SWS ( $\mathrm{p}=0.047)$. As age increased, gastrocnemius SWS decreased. Gastrocnemius SWS was also significantly different for participants older than 35 compared to those younger than $35(\mathrm{p}=0.039)$. Subjects in the older group exhibited a lower average gastrocnemius SWS $(2.14$ and $1.81 \mathrm{~m} / \mathrm{s}$ for the right and left legs, respectively) compared to the younger group ( 2.38 and $2.33 \mathrm{~m} / \mathrm{s}$ for the right and left legs, respectively).

There were no significant positive or negative correlations when age was compared to soleus SWS ( $\mathrm{p}=0.361)$ or Achilles SWS $(p=0.127)$. There were also no significant differences for soleus SWS ( $p=0.11$ ) or Achilles SWS ( $p=$ 0.744 ) when participants over 35 years old were compared to participants under 35 years old. The data pertaining to age can be seen in figure 2 .

\section{Tennis experience}

There was no significant positive or negative trend between the number of years that an athlete played tennis and gastrocnemius SWS ( $p=0.747)$, soleus SWS ( $p=0.257)$, or Achilles tendon SWS ( $\mathrm{p}=0.241)$. We also found no significant difference between SWS of tennis players with greater than or equal to 10 years of experience compared to those with less than 10 years of experience for the gastrocnemius $(\mathrm{p}=$ $0.380)$, soleus ( $\mathrm{p}=0.287)$, or Achilles tendon $(\mathrm{p}=0.311)$.

There was no significant positive or negative trend between the frequency of tennis play per week and gastrocnemius SWS ( $p=0.248)$, soleus SWS $(\mathrm{p}=0.725)$, or Achilles tendon SWS ( $\mathrm{p}=0.363)$. When comparing players' cumulative number of years of playing greater than 3 times per week to tissue SWS, no significant trend was observed for gastrocnemius $(\mathrm{p}=0.810)$, soleus $(\mathrm{p}=0.280)$, or Achilles tendon $(\mathrm{p}=0.968)$

\section{Effect of match timing}

Match timing had no significant effect on elastographic properties of the gastrocnemius $(\mathrm{p}=0.446)$, soleus $(\mathrm{p}=$ $0.834)$, or Achilles tendon $(\mathrm{p}=0.663)$.

\section{Other sports}

There was a significant difference between gastrocnemius SWS of the right leg versus the left leg depending on wheth- 


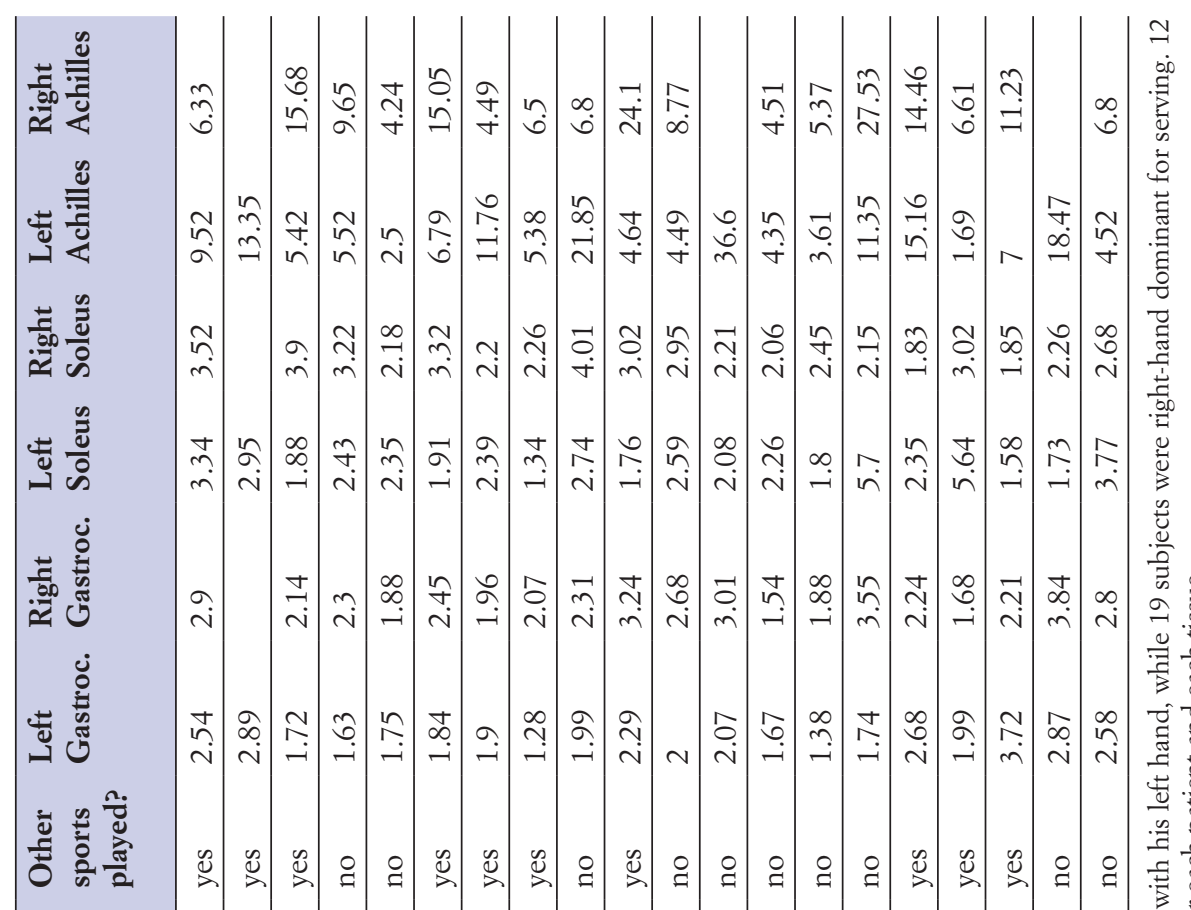

घ.

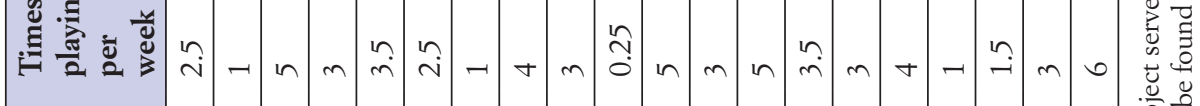

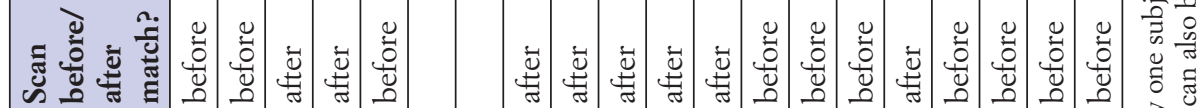

एदิ

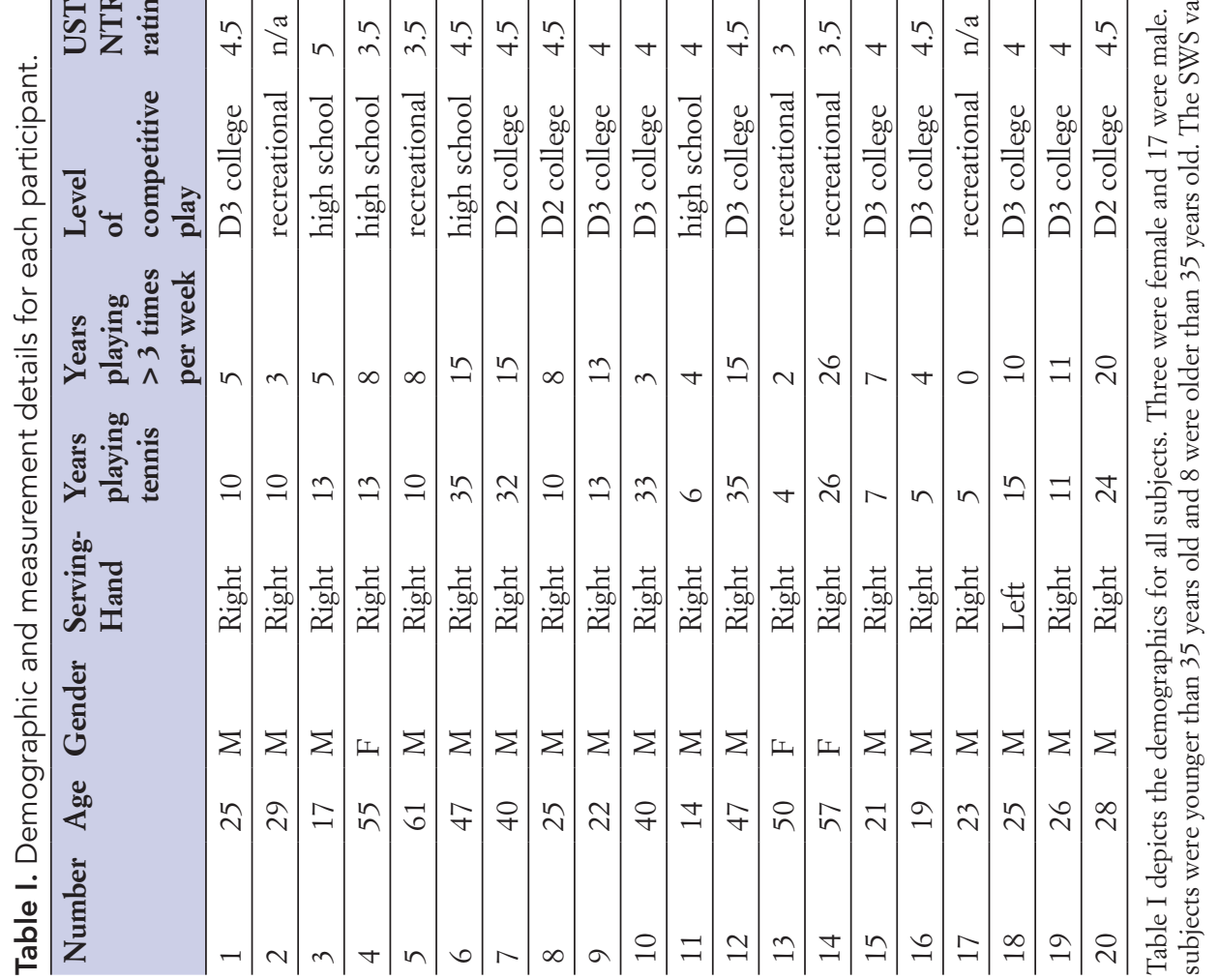


Table II. Organized demographics of participants.

\begin{tabular}{llllll}
\hline \multicolumn{7}{l}{ Highest } & Lowest & Average & & \\
\hline Age & 61 & 14 & 33.6 & Male & 17 \\
\hline Total years & 35 & 4 & 15.9 & Female & 3 \\
\hline Years $>$ 3 times/week & 26 & 0 & 9.1 & Recreational & 5 \\
\hline Gastrocnemius SWS & 3.84 & 1.28 & 2.29 & High School & 4 \\
\hline Soleus SWS & 5.70 & 1.34 & 2.66 & D3 college & 8 \\
\hline Achilles SWS & 36.60 & 1.69 & 10.06 & D2 college & 3 \\
\hline
\end{tabular}

Table II gives an organized picture of the demographics that were collected. The average age was 33.6 years old, and the average number of years that a participant played tennis was 15.9 years. The average SWS values for each tissue are also shown.

Table III. Summary of data.

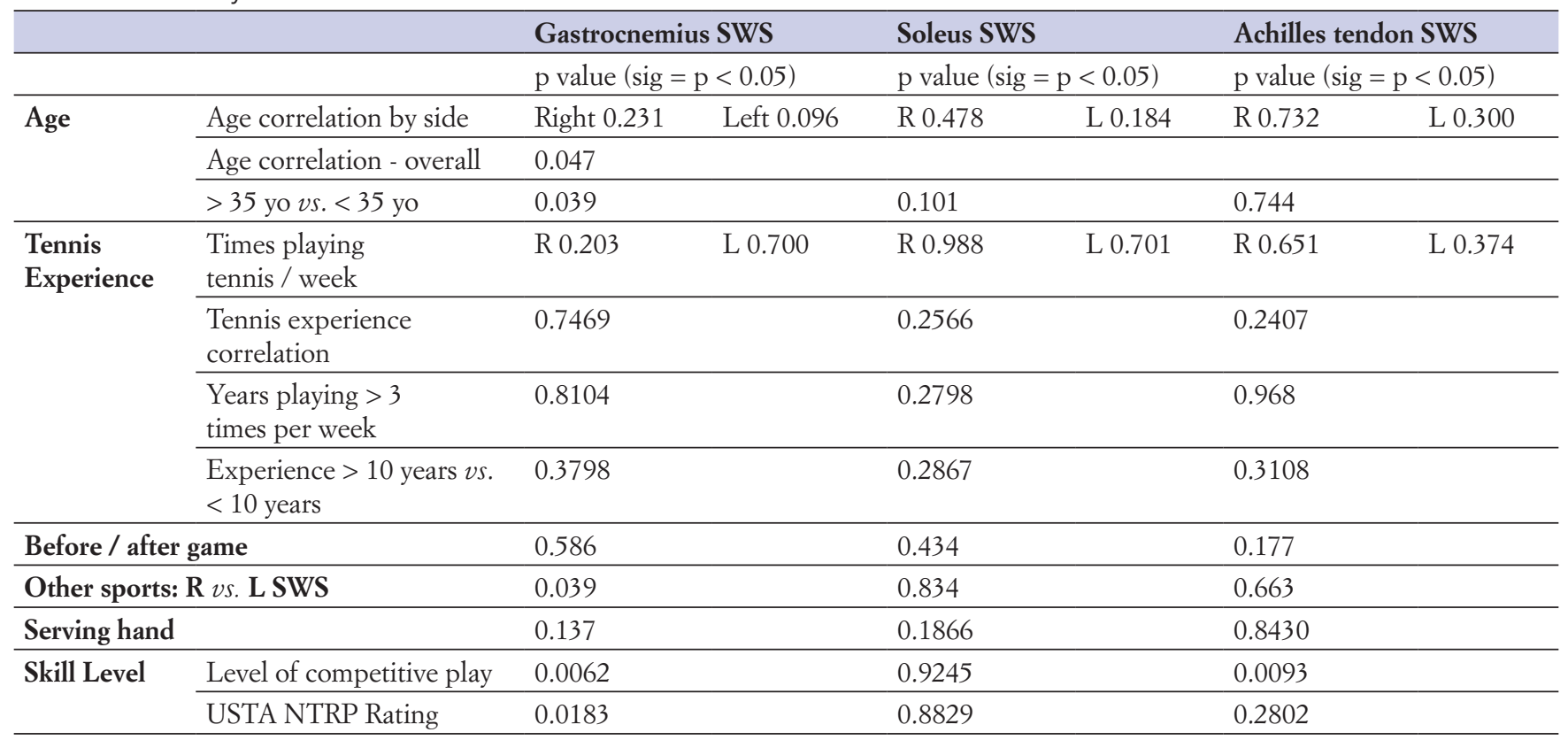

Table III summarizes the data that was analyzed for all three tissues compared to each demographic. Gastrocnemius SWS was significantly correlated with age and was significantly different for age $>35$ years old $v$ s. age $<35$ years old, whether or not other sports were played, and both skill level metrics. Soleus SWS was not found to significantly correlate with any demographics. Achilles tendon SWS was significantly different compared to the level of competitive play.

er tennis players played other sports $(\mathrm{p}=0.039)$. For tennis players who did not play other sports, gastrocnemius SWS ranged from (1.54-3.84) in the right leg and (1.38-2.87) in the left leg. For tennis players who did play other sports, gastrocnemius SWS ranged from (1.68-3.24) in the right leg and (1.28-2.89) in the left leg. These trends can be seen in figure 3.

No significant difference was identified between soleus SWS ( $p=0.834)$ or Achilles tendon SWS $(p=0.663)$ of the right versus left leg between participants who played only tennis when compared to multi-sport participants.

\section{Serving hand}

There was no significant trend between serving handedness and SWS of a player's right leg compared to their left leg for any tissue analyzed; gastrocnemius $(\mathrm{p}=0.137)$, soleus $(\mathrm{p}=$ $0.187)$, or Achilles tendon ( $\mathrm{p}=0.843)$.

\section{Skill level}

Interestingly, a tennis player's highest level of competitive play had a significant trend with gastrocnemius SWS ( $\mathrm{p}=$ 0.006 ) and Achilles tendon SWS ( $p=0.010)$, but not with 
Gastrocnemius SWS vs. age

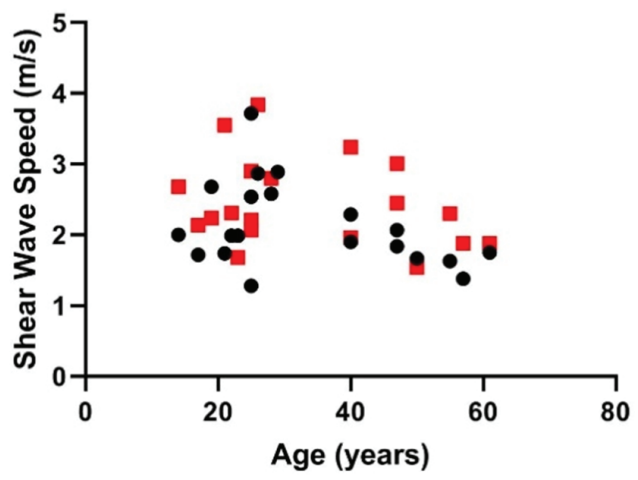

Gastrocnemius SWS vs. age groups

- Left

- Right

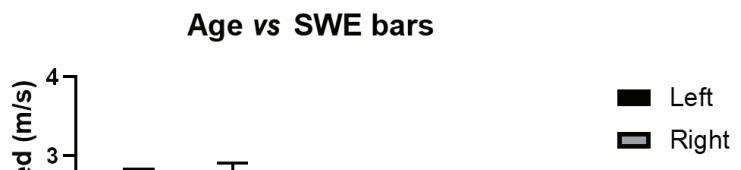

Figure 2. Left and right gastrocnemius SWS compared to age. Gastrocnemius SWS was significantly correlated with age ( $p=0.047)$ and was significantly different for participants above 35 years old compared to those less than 35 years old ( $p=0.039$ ). There was no significant difference in gastrocnemius SWS between legs (right leg $p=0.231$; left leg $p=0.096$ ). However, gastrocnemius SWS was significantly different in participants older than 35 years old compared to those younger than 35 years old ( $p=0.039$ ).

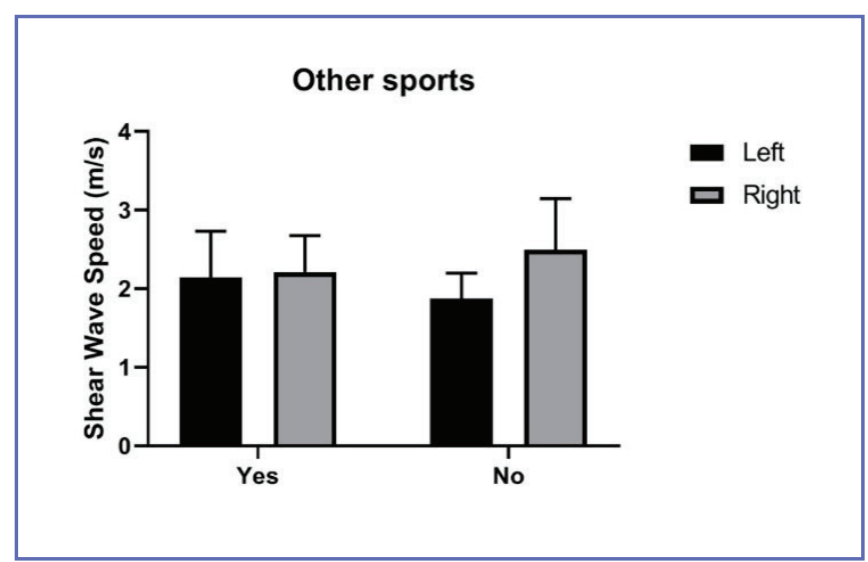

Figure 3. Left and right gastrocnemius SWS compared to whether the subject played another sport or tennis exclusively. Gastrocnemius SWS was significantly different between legs if the participant did not play other sports, but not if the participant did play other sports $(p=0.039)$. For participants who played other sports, the average gastrocnemius SWS was $2.32 \mathrm{~m} / \mathrm{s}$ for the right and $2.28 \mathrm{~m} / \mathrm{s}$ for the left. For participants who did not play other sports, gastrocnemius SWS was $2.57 \mathrm{~m} / \mathrm{s}$ for the right and $1.96 \mathrm{~m} / \mathrm{s}$ for the left.

soleus SWS ( $\mathrm{p}=0.925)$. Gastrocnemius SWS ranged from (1.38-2.89) for recreational tennis players, and (1.74-3.84) for college tennis players. Achilles tendon SWS ranged from (1.69-13.35) for recreational tennis players, and (4.64-36.6) for college tennis players.
A tennis player's USTA NTRP rating also significantly correlated with gastrocnemius SWS ( $p=0.180)$, but not for soleus SWS ( $\mathrm{p}=0.883)$ or Achilles tendon SWS $(\mathrm{p}=0.280)$.

\section{DISCUSSION}

This study quantified tissue characteristics in tennis players of varying tennis backgrounds to identify differences in elastographic properties between demographics. SWE was carried out on healthy tennis participants of an annual local tennis tournament either before or after match play. The gastrocnemius muscle was the only tissue that was found to have significant correlations with multiple variables. Achilles tendon SWS was found to be significantly different based on a player's skill level metrics. Soleus SWS was not found to have significant associations with any of the demographics, despite following a similar pattern seen in gastrocnemius SWS. SWE proved to be an effective technique to measure elastographic properties of the medial gastrocnemius musculotendinous junction.

Compared to the other tissues of the medial gastrocnemius musculotendinous junction, the gastrocnemius was most significantly affected by the metrics that were studied. This suggests that the gastrocnemius muscle is more likely to be affected by age and by movements performed in tennis compared to the soleus muscle or the Achilles tendon. Gastrocnemius SWS was also significantly affected by both metrics that measured a tennis player's skill level: highest level of competitive play and USTA NTRP rating. The high 
involvement of the gastrocnemius was also seen in a study by Delgado (14), where ultrasonography was used to identify the precise etiology of 141 patients' tennis leg symptoms (14). Of the cases they analyzed, $66 \%$ were caused by rupture of the medial gastrocnemius, $21 \%$ by joint involvement of the medial gastrocnemius and soleus, and only $0.7 \%$ were due to rupture of the soleus alone.

Our data shows that as age increased, gastrocnemius SWS significantly decreased. Additionally, gastrocnemius SWS was significantly lower in participants over 35 years old compared to those under 35 years of age, indicating that older aged players exhibited significantly less gastrocnemius tissue stiffness compared to younger tennis players. A similar result was observed by Yoshida (15), whereby SWE of the medial gastrocnemius musculotendinous junction resulted in greater elastic moduli in younger participants compared to older participants (15). Their participants were not specifically tennis players, so the observed negative correlation between gastrocnemius SWS and age is seen in populations other than tennis players. This negative correlation between age and gastrocnemius SWS can potentially be a factor related to injury. Calf strain occurs more frequently in older athletes compared to their younger counterparts (16). From a biomechanical point of view, tissues with lower mechanical strength are more likely to fail when loaded, which may explain the higher incidence of gastrocnemius tears compared to the Achilles tendon or soleus muscle. However, this contradicts conventional wisdom that considers musculotendinous stiffness a risk factor for musculoskeletal injury $(17,18)$. Younger individuals tend to exhibit lower joint stiffness and muscle tightness $(19,20)$. However, joint stiffness and muscle tightness are the result of the interaction of muscle with connective tissues such as tendons and ligaments. Aging affects each of those tissues differently, with tendons and ligaments exhibiting higher stiffness in older individuals (21). Therefore, it is possible that although muscle properties (shear modulus or SWS) decrease with age, joint stiffness and muscle tightness increase due to the stiffening of tendons and ligaments. Additionally, a review article from Murphy (17) reveals that many characteristics that are generally thought of as risk factors actually are not significantly correlated to risk (17). Our study demonstrated a positive association between each of the two skill level metrics and gastrocnemius SWS, but not between any of the four tennis experience metrics and gastrocnemius SWS. Achilles tendon SWS also exhibited an increasing trend with a player's highest level of competitive play. It is counterintuitive that gastrocnemius stiffness would significantly increase with increasing skill level, but lack a significant relationship when compared to experience; it is likely that players competing at a high skill level had to gain significant experience in the sport to reach that level and may not currently be playing as much tennis as they had in the past. Furthermore, extended low level tennis experience does not require these tissues to undergo the same changes as engagement at a high level of intensity. This is in accordance with studies investigating the relationship between muscle strength and elastic properties. Lima (22) used elastography to compare elastic properties of the gastrocnemius and soleus muscles to force generated by them (22). They concluded that elasticity of these muscles does not correlate to force. According to their conclusions, the demographic factors that we found to significantly correlate with increased stiffness do not relate to the force that can be generated by those tissues. Tennis involves eccentric, concentric, and isometric contractions; for example, Rafael (23) found that there is significant isometric contraction of the serratus anterior during tennis serve, forehand, and backhand swings (23). Gatz (24) found a significant difference in stiffness of the Achilles after a rehabilitation program limited to eccentric exercises, compared to a program that included eccentric and isometric movements (24). In their study, eccentric exercises yielded stiffer Achilles SWS measurements after a 12-week rehabilitation program for Achilles strain, while a combination of eccentric and isometric maneuvers did not produce significant changes in tissue stiffness. Tennis players who exhibit demographics that correlate with stiffer gastrocnemius tissue could consider incorporating isometric movements in their routines to mitigate further tissue stiffening. However, more research correlating isometric exercise, calf muscle stiffness, and calf strain needs to be conducted to study the efficacy of such exercise.

To our knowledge, there have been no other studies that analyzed structural tissue changes in comparison to the skill level of tennis players. However, a study conducted by Agresta (25) investigated how a runner's "stride-to-stride fluctuations" can adapt to changing demands based on the runner's length of experience in the sport (25). They found that increased experience allowed runners to modify gait more skillfully in response to changing demands. Experience alone was enough to correlate with significant changes in a runner's stride. In contrast, our study found that experience alone was not sufficient to elicit significant changes in tissue stiffness.

\section{Limitations}

The multiple comparison issue arises when looking at multiple variables simultaneously, which could result in false positives. The multiple effects model was chosen to carry out data analysis to combat this phenomenon. The small sample size of the study is itself a limitation, but this does not affect the significance of the results due to the method of 
analysis being the mixed effects model. Further, all subjects were of male gender, which keeps the sample homogenous. It is a good representation of the male tennis population because of the wide age range of participants.

\section{CONCLUSIONS}

In conclusion, SWE was used to measure elastographic properties of the tissues comprising the medial musculotendinous junction of the Achilles tendon in tennis players. Age, exclusively playing tennis as opposed to other sports, and two metrics of skill level (highest level of competitive play and USTA NTRP rating) significantly affected gastrocnemius SWS. Highest level of competitive play was the only metric found to affect Achilles tendon SWS. Soleus SWS exhibited no significant changes with any of the variables, despite following similar trends seen with gastrocnemius SWS. The results suggest that the gastrocnemius muscle is disproportionately involved in the movements conducted in tennis compared to the other tissues comprising the medial Achilles musculotendinous junction.

\section{REFERENCES}

1. Domeracki SJ, Landman Z, Blanc PD, Guntur S. Off the Courts: Occupational "Tennis Leg". Workplace Health Saf 2019;67(1):5-8.

2. Gescheit DT, Cormack SJ, Duffield R, et al. Injury epidemiology of tennis players at the 2011-2016 Australian Open Grand Slam. Br J Sports Med 2017;51(17):128994.

3. Miller WA. Rupture of the musculotendinous juncture of the medial head of the gastrocnemius muscle. Am J Sports Med 1977;5(5):191-3.

4. Powell RW. Lawn Tennis Leg. Lancet 1883;122(3123):44.

5. Shah JR, Shah BR, Shah AB. Pictorial essay: Ultrasonography in 'tennis leg'. Indian J Radiol Imaging 2010;20(4):269-73.

6. Petruska A. Calf Strain. Boston Sports Medicine \& Research Institute. Available at: www.bostonsportsmedicine.com. Last access date: 03/17/2019.

7. Kubo K, Miyazaki D, Tanaka S, Shimoju S, Tsunoda N. Relationship between Achilles tendon properties and foot strike patterns in long-distance runners. J Sports Sci 2015;33(7):665-9.

8. Balaban M, Idilman IS, Ipek A, Ikiz SS, Bektaser B, Gumus M. Elastographic findings of achilles tendons in asymptomatic professional male volleyball players. J Ultrasound Med 2016;35(12):2623-8.

9. Padulo J, Oliva F, Frizziero A, Maffulli N. Basic principles and recommendations in clinical and field science research: 2018 update. Muscles Ligaments Tendons J 2018;8(3):305-7.

10. Petitpierre F, Perez J-T, Bise S, Fournier C, Hauger O, Dallaudière B. Quantitative elastography of Achilles tendon using Shear Wave Elastography (SWE): correlation with zonal anatomy. Muscles Ligaments Tendons J 2019;08(02):229.
The negative correlation between age and SWS highlights the ambiguous, inconsistent relationship between tissue stiffness and vulnerability to injury, as other researchers have shown. Future studies could analyze a larger cohort of individuals for greater statistical power or analyze an all-female sample to compare how the results could differ by gender.

\section{ACKNOWLEDGEMENTS}

We thank Andrea Horne for managing IRB submissions and for helping to set up for data collection.

\section{CONFLICT OF INTERESTS}

The authors declare that they have no conflict of interests. Robert A. Gallo, M.D., M.H.A., is a member of the advisory board of Kalibur Labs, editorial board for Current Reviews in Musculoskeletal Medicine and Sports Medicine and Arthroscopy Reviews, and is member of Public Relations Committee of American Orthopaedic Society for Sports Medicine.

11. Bercoff J, Tanter M, Fink M. Supersonic shear imaging: A new technique for soft tissue elasticity mapping. IEEE Trans Ultrason Ferroelectr Freq Control 2004;51(4):396-409.

12. Lin C-Y, Sadeghi S, Bader DA, Cortes DH. Ultrasound Shear Wave Elastography of the Elbow Ulnar Collateral Ligament: Reliability Test and a Preliminary Case Study in a Baseball Pitcher. J Eng Sci Med Diagnostics Ther 2018;1(1):011004.

13. Sadeghi S, Newman C, Cortes DH. Change in skeletal muscle stiffness after running competition is dependent on both running distance and recovery time: A pilot study. PeerJ 2018;6:e4469.

14. Delgado GJ, Chung CB, Lektrakul N, et al. Tennis leg: Clinical US study of 141 patients and anatomic investigation of four cadavers with MR imaging and US. Radiology 2002;224(1):112-9.

15. Yoshida K, Itoigawa Y, Maruyama Y, et al. Application of shear wave elastography for the gastrocnemius medial head to tennis leg. Clin Anat 2017;30(1):114-9.

16. Green B, Pizzari T. Calf muscle strain injuries in sport: a systematic review of risk factors for injury. Br J Sports Med 2017;51(16):1189-94.

17. Murphy DF, Connolly DA, Beynnon BD. Risk factors for lower extremity injury: a review of the literature. Br J Sports Med 2003;37(1):13-29.

18. Krivickas LS, Feinberg JH. Lower extremity injuries in college athletes: Relation between ligamentous laxity and lower extremity muscle tightness. Arch Phys Med Rehabil 1996;77(11):1139-43.

19. Lorimer AV, Hume PA. Stiffness as a Risk Factor for Achilles Tendon Injury in Running Athletes. Sports Med 2016;46(12):1921-38. 
20. Hrysomallis C. Injury incidence, risk factors and prevention in Australian rules football. Sports Med 2013;43(5):339-54.

21. Delabastita T, Bogaerts S, Vanwanseele B. Age-Related Changes in Achilles Tendon Stiffness and Impact on Functional Activities: A Systematic Review and Meta-Analysis. J Aging Phys Act 2018:1-12. Epub ahead of print.

22. Lima K, Martins N, Pereira W, Oliveira L. Triceps surae elasticity modulus measured by shear wave elastography is not correlated to the plantar flexion torque. Muscles Ligaments Tendons J 2017;7(2):347-52.
23. Escamilla RF, Andrews JR. Shoulder muscle recruitment patterns and related biomechanics during upper extremity Sports Med 2009;39(7):569-90.

24. Gatz M, Betsch M, Tingart M, et al. Effect of a 12-week eccentric and isometric training in achilles tendinopathy on the gastrocnemius muscle: An ultrasound shear wave elastography study. Muscles Ligaments Tendons J 2020;10(1):92-9.

25. Agresta CE, Goulet GC, Peacock J, Housner J, Zernicke RF, Zendler JD. Years of running experience influences stride-tostride fluctuations and adaptive response during step frequency perturbations in healthy distance runners. Gait Posture 2019;70:376-82. 